\title{
Hyperspectral Data Restoration in Synchrotron-based Infrared Microspectroscopic Imaging
}

\author{
E. Mattson*, M. Nasse***, and C. J. Hirschmug1* \\ *Dept. of Physics, University of Wisconsin-Milwaukee, Milwaukee, WI 53211 \\ **Synchrotron Radiation Center, University of Wisconsin-Madison, Stoughton, WI 53589
}

Infrared Microspectroscopy (IRMS), a technique that combines the chemical specificity of IR spectroscopy with the spatially resolved information of microscopy, has traditionally been limited by a fundamental tradeoff between data acquisition time, SNR, and spatial resolution . A new IR beamline at the Synchrotron Radiation Center, Infrared Environmental Imaging (IRENI) [1], has been designed and commissioned to overcome these challenges through oversampling with a focal plane array detector, thus collecting diffraction limited IR images with high SNR with minimal acquisition time. However, by the nature of diffraction-limited resolution, every measured image is a convolution of the true image with the Point Spread Function (PSF) of the optical system. Consequently, the stack of digital absorbance images (i.e. Hyperspectral cube) that comprise the spatially resolved IR spectra is blurred, resulting in a weighted spatial averaging of every spectrum. In order to produce spectra that reflect optimal spatial resolution, we have characterized the wavelength-dependent PSF of the Bruker Vertex 70 IR microscope equipped with a 74x objective (Fig. 1) to implement well-established deconvolution algorithms. The PSF was measured by imaging IR transmittance of 2, 3, and 5 micron pinholes to obtain its complete mid-IR wavelength dependence. The transmittance images were fit to a diffraction pattern of an annulus as a simple analytical model of the mirrored Schwarzschild optics. Knowing the wavelength-dependent PSF enabled the deconvolution of every IR image in a hyperspectral cube. By reassembling the stack of deconvolved images, restored images and spectra were recovered over the entire mid-IR spectral range. Restoration methods including Fourier filtering and Total Variation (TV) regularization were implemented.

Test samples including USAF resolving test charts and polystyrene (PS) spheres embedded in a Polyurethane (PU) film were examined. Deconvoluted images of the USAF chart at two wavelengths indicate that deconvolution algorithms enhance image contrast and preserve resolution at or above the diffraction limit (Figs. $2 \mathrm{a}$ and b). Figure 3a shows absorbance images of PS spheres embedded in PU produced from C-H stretch features. Reconstructed spectra (Fig. 3d) from points progressing across the interface of the two beads show varying absorbance of the PS and PU features in the $\mathrm{C}-\mathrm{H}$ stretch region, reflecting the changing quantities of PS and PU across the interface. No distinction is observed in the original spectra (Fig. 3c). Thus, implementation of PSF deconvolution over the entire mid-IR spectral region produces superior spatial resolution as well as enhanced spectral sensitivity at chemical interfaces. 
References:

[1] Nasse, M.J., et al., Nucl. Instrum. Methods Phys. Res. Sec. A. 582 (2006) 1

[2] This work is funded under NSF Award No. DMR-0619759 and DMR-0537588
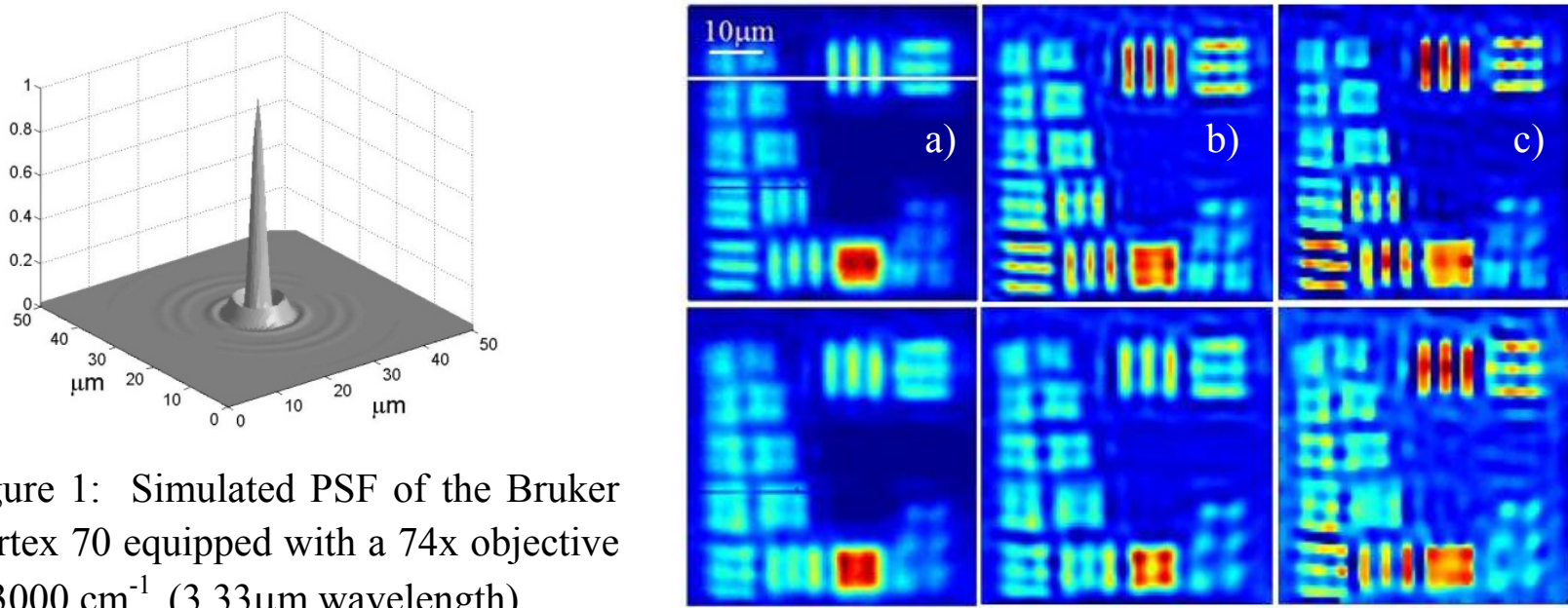

Figure 1: Simulated PSF of the Bruker Vertex 70 equipped with a $74 x$ objective at $3000 \mathrm{~cm}^{-1}$ (3.33 $\mathrm{mm}$ wavelength)

Figure 2: USAF 3-bar resolving test chart at $3000 \mathrm{~cm}-1$ (top) $2500 \mathrm{~cm}-1$ (bottom). Original images are shown in (a) with deconvolution by Fourier Filtering (b) and TV regularization (c). Profiles through the indicated line are shown for $3000 \mathrm{~cm}^{-1}$ in (d) and $2500 \mathrm{~cm}^{-1}$ in (e). Both methods of image restoration enhance contrast and resolution with minimal artifacts left at jump
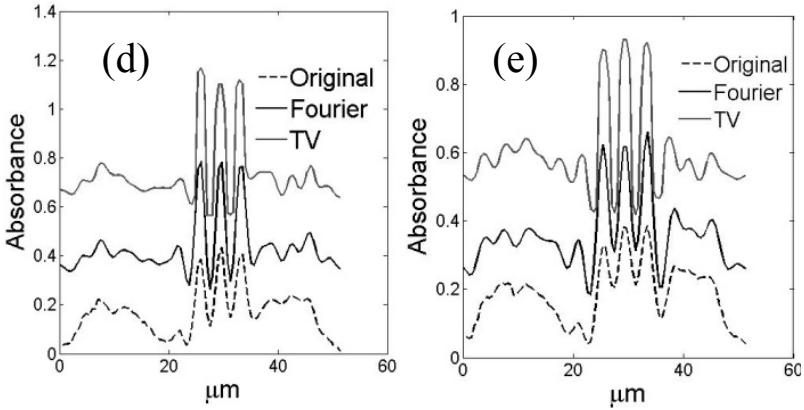
discontinuities.
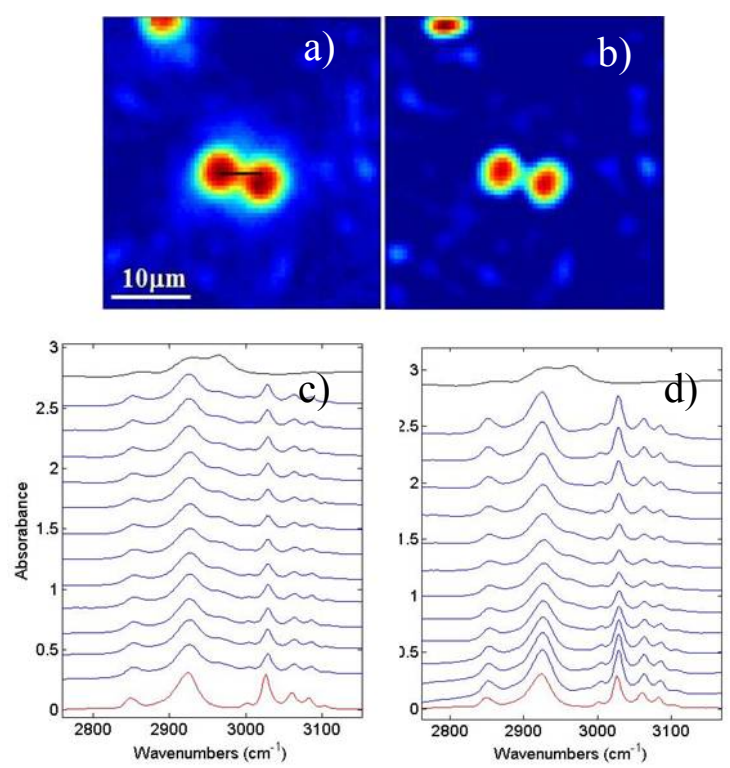

Figure 3: Original (a) and restored (b) images of PS beads embedded in PU generated by integrating over 2900-3000 $\mathrm{cm}^{-1}$. The black line in (a) indicates the region over which the original (c) and reconstructed (d) spectra were taken from. In (c) and (d), the bottom and top spectra correspond to PS and PU standards, respectively. Other spectra, from bottom to top, move from left to right along the black line. In (d), PS absorptions are strongest in the center of the beads and decrease at the interface, while PU absorptions are strongest at the interface and weakest on top of the beads. Such distinctions are not present in (c). 